\title{
Nonlinear Landau-Zener Tunnelling in Coupled Waveguide Arrays
}

\author{
Ramaz Khomeriki \\ Physics Department, Tbilisi State University, 3 Chavchavadze, 0128 Tbilisi (Georgia) \\ Max-Planck-Institut fur Physik komplexer Systeme, Nöthnitzer str. 38, 01187 Dresden (Germany)
}

\begin{abstract}
The possibility of direct observation of Nonlinear Landau-Zener tunnelling effect with a device consisting of two waveguide arrays connected with a tilted reduced refractive index barrier is discussed. Numerical simulations on this realistic setup are interpreted via simplified double well system and different asymmetric tunnelling scenarios were predicted just varying injected beam intensity.
\end{abstract}

PACS numbers: 42.65.Wi, 42.82.Et, 03.65.w, 05.45.-a

Landau-Zener tunnelling effect [1] has been first proposed for interpretation of atomic level mixing in predissociasion process [2]. More recently this model has been applied to explain transitions between Bloch modes in periodic systems, particularly for Bose-Einstein Condensates (BEC) [3, 4] and acoustic waves in layered and elastic structures [5]. Later on the same effect of Bloch mode transitions has been investigated in optical systems with variety of architectures: waveguide arrays with a step in a refractive index [6], arrays with applied temperature gradient [7], curved waveguides [8], nematic crystals [9] and two dimensional photonic lattices [10], among others.

The nonlinear extension of Landau-Zener model is first analyzed theoretically [11, 12] in case of BEC in optical lattices and asymmetric transition processes have been found in contrast to the linear limit. Later on this nontrivial behavior has been experimentally confirmed 14] and generalized Landau-Zener transition formula has been analytically derived [13]. In all of these previous studies the tunnelling processes between different Bloch modes are considered, while, in principle, the same Landau-Zener tunnelling effect should take place for double well system. Indeed, very recently the tunnelling scenarios between two spatial modes of BEC has been proposed [15, 16]. In the present paper we propose to use two coupled waveguide arrays with a tilted (with respect to the waveguide direction) reduced refractive index barrier for visual observation of asymmetric nonlinear tunnelling effects between the arrays.

The refraction index profile of a suggested experimental device is presented in insets of Fig. 1 and refractive index pattern could be realized either by microfabrication [17] or by laser beams in photonic lattices [10]. The beam is injected either into the left or right array and its intensity has a harmonic profile across the injection array (this is schematically represented as black arrows with different lengths in the insets). In case of small intensity (linear regime) the light injected into the left array tunnels to the right array and vice versa. Increasing the injected beam intensity the symmetry breaks down, particularly, injecting the beam at the left it again tunnels to the right array, while injecting the beam with the same intensity at the right it stays trapped there (see the corresponding graphs in Fig. 1). Further increase of the injected intensity leads to the beam trapping irrespective

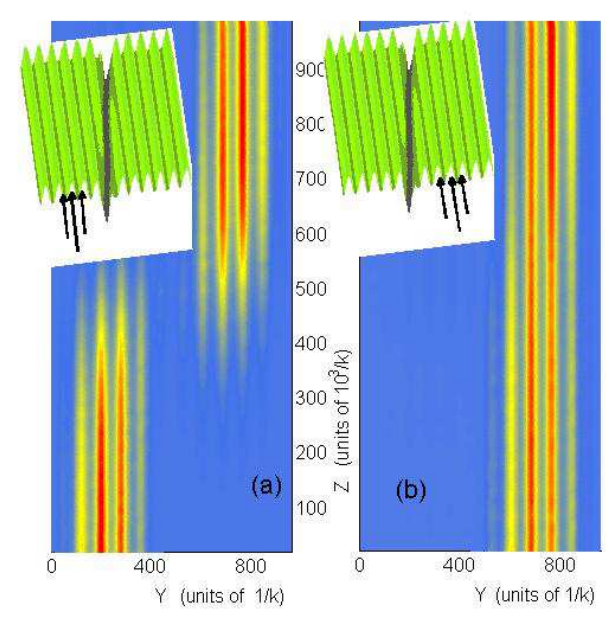

FIG. 1: (Color online) Graphs (a) and (b): results of numerical simulations (stationary spatial light intensity distribution) on the initial model (1) with the amplitude 0.0018 of injected light wave pattern $\Psi(Y, 0)$. Insets show the device schematics and corresponding injection positions.

to the place of the injection of the beam. Below I shall interpret this nontrivial effect via Nonlinear Landau-Zener tunnelling in a simple two degree of freedom system.

Let us start from writing a wave equation for linearly polarized electric field in paraxial approximation:

$$
i \frac{\partial \Psi}{\partial Z}+\frac{1}{2} \frac{\partial^{2} \Psi}{\partial Y^{2}}+\Delta(Y, Z) \Psi+|\Psi|^{2} \Psi=0,
$$

where I assume light propagation in nonmagnetic optical film ( $Y Z$ plane) along $Z$ direction, $\Psi(Y, Z)$ stands for a complex wave envelope, $\Delta(Y, Z)=\left(n-n_{0}\right) / n_{0}$ is a linear refractive index variation along $Y$ and $Z$ axis, the dimensionless spatial variables $Y$ and $Z$ are scaled in units of inverse carrier wavenumber $1 / k$, and this wavenumber is defined as $k=n_{0} \omega / c$ with $\omega$ being laser beam frequency and focusing Kerr nonlinearity is scaled to unity. Let us consider pinned boundary conditions (i.e. $\Psi(Y, Z)=0$ for $\left.Y=Y_{\min }, Y_{\max }\right)$, while periodic modulations together with tilted reduced refractive index barrier (see insets of Fig. 1) are modelled as follows:

$$
\Delta(Y, Z)=\delta n_{1} \sin ^{2}(K Y)-\delta n_{2} \operatorname{sech}[(\mathrm{Y}-\Gamma \mathrm{Z}) / \Lambda]
$$




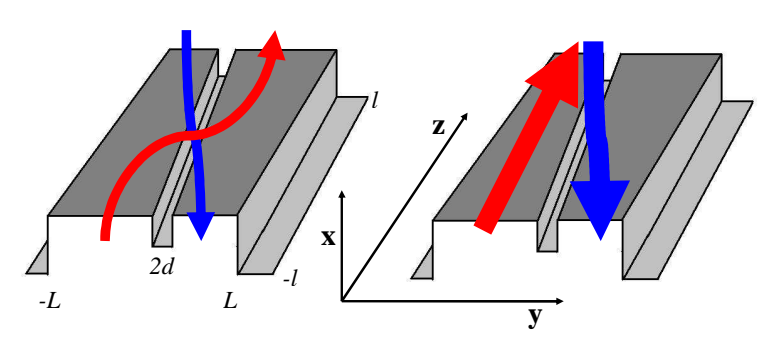

FIG. 2: (Color online) Reduction of the initial problem (1) with the refractive index profiles presented in the insets of Fig. 1 to the effective double well system with "moving" barrier. The curved lines describe the light intensity dynamics for the linear (left graph) and asymmetric nonlinear (right graph) regimes.

$\delta n_{1}$ and $\delta n_{2}$ stand for the amplitudes of periodic (scaled by $K$ ) modulation and barrier, respectively, while $\Gamma$ and $\Lambda$ define a tilt angle and a width of the reduced refractive index barrier. In numerical simulations on the model (1) the following values are fixed: $\delta n_{1}=0.002$ and $\delta n_{2}=$ 0.003 .

As well established, the problem of periodic array of effective waveguides could be simplified via tight binding discretization procedure [18, 19] when one can present the envelope wavefunction $\Psi(y, z)$ as an expansion over approximate gaussian eigenmodes of individual waveguides:

$\Psi(Y, Z)=\sum_{j} \mathcal{E}_{j}(Z) \phi_{j}(Y) \quad \phi_{j}(Y) \sim e^{-K \sqrt{\delta n_{1} / 8}\left(Y-R_{j}\right)^{2}}$

where $j$ numbers waveguide center positions and thus $R_{j}=\pi(2 j+1) / 2 K$. Then one gets a Discrete Nonlinear Schrödinger (DNLS) equation representation of the problem:

$$
i \frac{\partial \mathcal{E}_{j}}{\partial Z}+\frac{C}{2}\left(\mathcal{E}_{j+1}+\mathcal{E}_{j-1}\right)-V(j, z) \mathcal{E}_{j}+\chi\left|\mathcal{E}_{j}\right|^{2} \mathcal{E}_{j}=0,
$$

where coupling constant $C$ is calculated from the overlapping integrals between neighboring waveguide eigenmodes, while $\chi$ counts only the nonlinear overlap integral of the single eigenmode. Effective potential barrier $V(j, Z)$ could be approximated as (see Ref. [20]):

$$
V(j, Z)=V_{0} \operatorname{sech}[(\pi \mathrm{j}-\Gamma \mathrm{KZ}) / \mathrm{K} \Lambda],
$$

where $V_{0} \sim \delta n_{2}$ is a potential barrier height. Further reduction of (3) is made associating $j$ with a continuous new spatial variable $y=Y K / \pi$ and then defining $z=$ $C Z$ and rescaling $\mathcal{E} \rightarrow \mathcal{E} \sqrt{\chi / C} \exp [i C Z], V \rightarrow V / C$ one gets Nonlinear Scrödinger (NLS) equation in an external double well potential with "moving" barrier:

$$
i \frac{\partial \mathcal{E}}{\partial z}+\frac{1}{2} \frac{\partial^{2} \mathcal{E}}{\partial y^{2}}-V(y, z) \mathcal{E}+|\mathcal{E}|^{2} \mathcal{E}=0 .
$$

The meaning of discretization of initial equation (10) getting DNLS (31) and then subsequent continuous approximation to NLS equation (5) is that we get rid of periodical modulation of refractive index which are present in initial equation (11). Indeed, in NLS (5) one is left only with "moving" barrier potential and the problem is reduced to the two coupled waveguides case (see Fig. 2). Our aim is to give analytical consideration of the latter problem and present the interpretation of the numerical simulations undertaken on the initial equation (11).

For the clarity of presentation let us choose symmetric boundaries $-L<y<L$ and $-\ell_{0}<z<\ell$ and we shall find the solutions of (5) in case of pinned boundary conditions $\mathcal{E}(-L, z)=\mathcal{E}(L, z)=0$. Moreover, we will require small value for the barrier tilt parameter $\Gamma \rightarrow 0$ and then expanding expression for the barrier (4) over small parameter $\Gamma z$ we get

$$
V(y, z)=V_{s}(y)+\Gamma z V_{t}(y)
$$

Thus the potential barrier expression is split into symmetric $V_{s}(y)$ and antisymmetric $V_{t}(y)$ parts with respect to the inversion transformation $y \rightarrow-y$ :

$$
V_{s}(y)=\frac{V_{0}}{\cosh [\pi y / K \Lambda]} \quad V_{t}(y)=\frac{V_{0} \sinh [\pi y / K \Lambda]}{\Lambda \cosh ^{2}[\pi y / K \Lambda]}
$$

and let us build the stationary solutions of (5) with potential $V_{s}(y)$ considering the second term $V_{t}(y)$ as a perturbation. Particularly, in the zero approximation we are left with the problem of symmetric double well potential which has two lowest eigenvalue symmetric $\Phi^{+}(y)$ and antisymmetric $\Phi^{-}(y)$ orthonormalized solutions and one can simply construct from them two functions

$$
\phi_{1}=\left(\Phi^{+}+\Phi^{-}\right) / \sqrt{2}, \quad \phi_{2}=\left(\Phi^{+}-\Phi^{-}\right) / \sqrt{2}
$$

localized at the left and right wells, respectively.

Then one can separate the variables in $\mathcal{E}(y, z)$ establishing a dimer model as

$$
\mathcal{E}(y, z)=\psi_{1}(z) \phi_{1}(y)+\psi_{2}(z) \phi_{2}(y), \quad \psi_{1}^{2}+\psi_{2}^{2}=P_{t}
$$

and substituting this into (5) where potential function is taken in the form (6), multiplying on $\phi_{1}(y)$ and $\phi_{2}(y)$, then integrating over $y$ and discarding common phase variables we recover the nonlinear Landau-Zener model [1] in its standard form:

$$
\begin{gathered}
-i \frac{\partial \psi_{1}}{\partial z}=\alpha z \psi_{1}+v \psi_{2}+r\left(\left|\psi_{1}\right|^{2}-\left|\psi_{2}\right|^{2}\right) \psi_{1}, \\
-i \frac{\partial \psi_{2}}{\partial z}=-\alpha z \psi_{2}+v \psi_{1}-r\left(\left|\psi_{1}\right|^{2}-\left|\psi_{2}\right|^{2}\right) \psi_{2} .
\end{gathered}
$$

where the parameters could be calculated as follows:

$2 r=\int_{-L}^{L} d y \phi_{1,2}^{4}, \quad v=\int_{-L}^{L} d y \phi_{1,2}\left(\frac{\partial^{2} \phi_{2,1}}{\partial y^{2}}-V_{s}(y) \phi_{2,1}\right)$ 

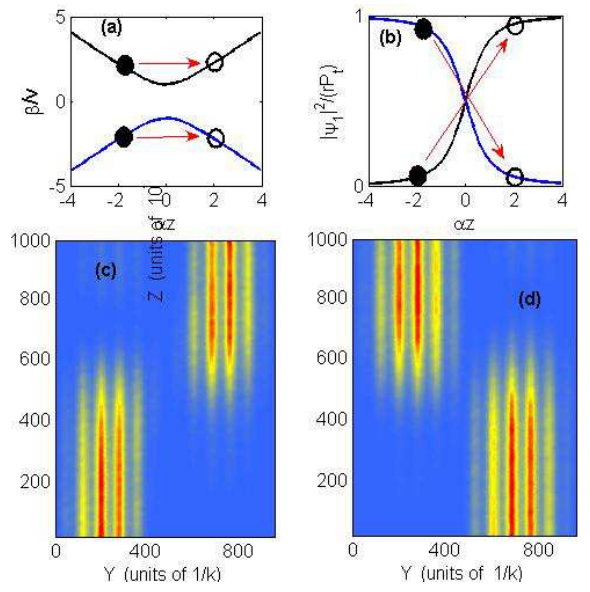

FIG. 3: (Color online) Graphs (a) and (b) display dependencies of the propagation constant $\beta$ and beam intensity at the left array $\left|\psi_{1}\right|^{2}$ on the parameter $\alpha z$ according to equation (11) in linear regime. Solid circles indicate initial states of the system and open circles stand for the final states in adiabatic regime. Lower graphs display the results of numerical simulations on the initial model equation (1) when small intensity light is injected into the left (graph c) and right (graph d) arrays, respectively.

and $\alpha=-\Gamma \int_{-L}^{L} d y \phi_{1,2} V_{t}(y) \phi_{1,2}$ is an effective "acceleration" parameter.

In the linear limit, i.e. when total intensity $\psi_{1}^{2}+\psi_{2}^{2}=$ $P_{t} \rightarrow 0$ the above equation is just an ordinary LandauZener tunnelling [1] which has a well known result that if "acceleration" $\alpha$ is large or coupling $v$ is small the light remains in the array where it was injected initially. Otherwise, in adiabatic limit, the light tunnels to other waveguide array, thus the picture is symmetric. In our

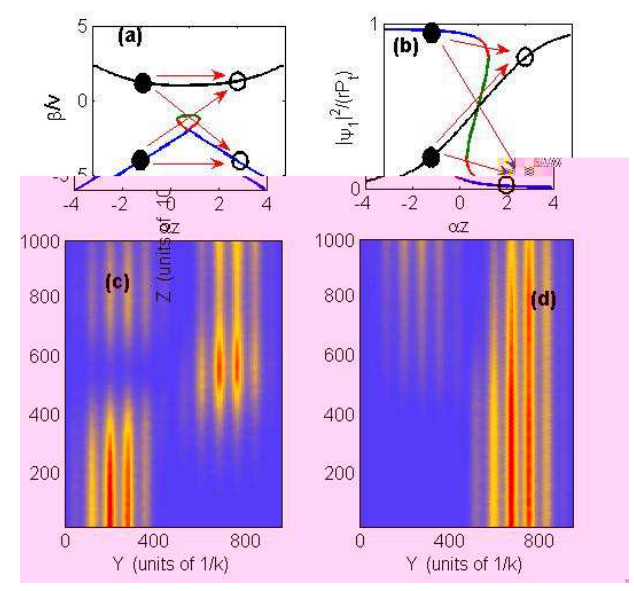

FIG. 4: (Color online) The same as in Fig. 3 but with different parameters: Upper graphs correspond to the intermediate range of effective nonlinearity $r P_{t}=1.5$. Lower graphs display the light intensity distribution in numerical simulations on initial equation (1) with wave envelope amplitude 0.0016 . numerical simulations on the initial model equation (1) the barrier tilt angle $\Gamma \sim \alpha$ is small guaranteing adiabaticity of the process and one should see tunnelling of the light irrespective to the beam injection place.

In order to clarify the behavior of the nonlinear two degree of freedom system (10) let us seek for the stationary solutions in the form $\psi_{1,2}=\left|\psi_{1,2}\right| \exp (i \beta z)$ getting thus a quartic equation for the propagation constant $\beta$ and dependencies of intensities $\left|\psi_{1}\right|^{2}$ and $\left|\psi_{2}\right|^{2}$ versus $\alpha z$ :

$$
\begin{aligned}
(\alpha z)^{2} \beta^{2} & =\left(\beta^{2}-v^{2}\right)\left(\beta+r P_{t}\right)^{2}, \\
2\left|\psi_{1}\right|^{2} & =r P_{t}\left(1+\frac{\alpha z}{\beta+r P_{t}}\right), \quad\left|\psi_{2}\right|^{2}=r P_{t}-\left|\psi_{1}\right|^{2} .
\end{aligned}
$$

From these it automatically follows that the equation for $\beta$ has four real roots if effective nonlinearity exceeds a coupling strength $r P_{t}>v$, otherwise it has two real roots for fixed $z$ (for more details see Ref. [11, 12]). The corresponding dependencies of $\beta$ and $\left|\psi_{1}\right|^{2}$ on $z$ for various total intensities are displayed in Figs. 3, 4 and 5 .
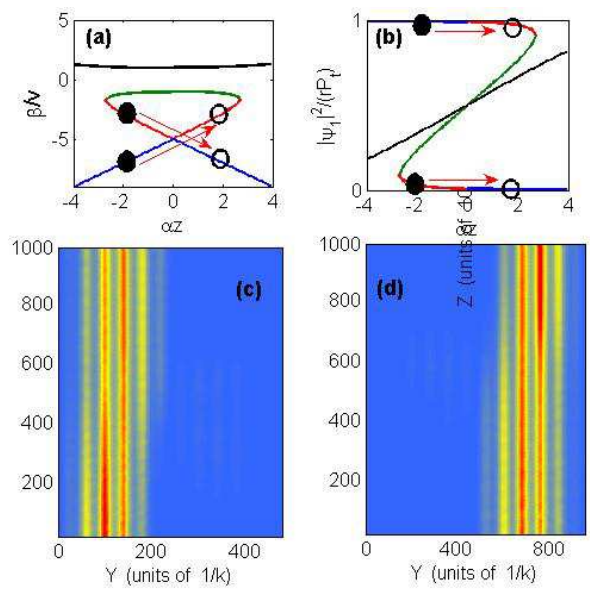

FIG. 5: (Color online) Upper graphs correspond to the strong effective nonlinearity range $r P_{t}>2$ and lower graphs display the light intensity distribution in numerical simulations with corresponding value of wave envelope amplitude 0.002 .

Let us first consider the linear regime $r P_{t} \rightarrow 0$ (see Fig. 3). Then the propagation constant is characterized by two solutions for fixed $z$. The solid circles in upper panels of Fig. 3 correspond to the starting point $z=-\ell$ and we choose $\alpha \ell=2$, when almost all the intensity is concentrated in the left waveguide array. This means that we should follow blue curve and in case of adiabatic process the system passes the point $z=0$ and propagation constant follows further the blue curve and as a result the system will end up with almost zero intensity at the left waveguide array, i.e. all the intensity should go into the right one. The same happens if the light is injected at the right waveguide array (in this case the system is initially on the black curve) and the light tunnels into the left array. Indeed, in numerical simulations (see bottom panel of Fig. 3) on the initial equation (1) 

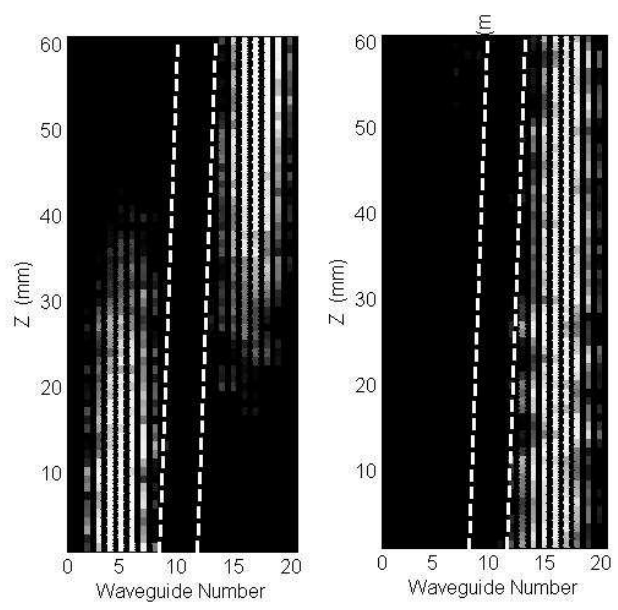

FIG. 6: Numerical simulations on the discretized model (3) with the parameters $C=100 \mathrm{~mm}^{-1}$ and $\chi=6.5 \mathrm{~mm}^{-1} W^{-1}$. The and height of the barrier and its width are $V_{\max }=$ $0.6 \mathrm{~mm}^{-1}$ and $\Lambda=25 \mu \mathrm{m}$. Dashed lines indicate position of tilted reduced refractive index barrier and peak intensity in both cases is $\left|\mathcal{E}_{j}(Z=0)\right|_{\max }^{2}=31 \mathrm{~W}$.

it appears that beam injection with harmonic profile and small amplitude into the left array leads to the tunnelling to the right array and vice versa.

Next let us consider the case when effective nonlinearity exceeds a coupling constant $r P_{t}>v$. First of all we note that as seen from the equations (11) a simple rescaling of all quantities with respect to the coupling constant $v$ is possible and thus in our further analysis we can set $v=1$ without loss of generality. Thus in case of effective nonlinearities $r P_{t}>1$ the propagation constant versus $\alpha z$ diagram acquires a butterfly structure (see upper panels of Fig. 4) and starting again from the point $\alpha z=-2$ and with almost whole intensity in the left waveguide array $\left(\left|\psi_{1}\right|^{2} \simeq r P_{t}\right)$ the evolution along $z$ follows the blue line (see both graphs of the upper panel of Fig. 4) then it passes to the red line region where the butterfly structure begins [we note that different colors in Fig. 4 corresponds to the four different solutions of quartic equation (11)]. By end of the red line there exists a discontinuity, thus the system has to jump either to the blue line or to the black one even in ideally adiabatic case. On the other hand, starting with zero intensity at the left waveguide array (all the intensity is concentrated at the right, i.e. $\left|\psi_{1}\right|^{2} \simeq 0$ ) the system follows the black line and continues safely until the end point $\alpha z=2$. This is the reason of asymmetric behavior of the nonlinear case, thus injecting the beam into different waveguide arrays one can end up at the same array. Different amplitudes of initial harmonic beam profiles have been checked for this tunnelling scenario. For instance, in numerical simulations displayed in lower panels of Fig. 4 the beam amplitude is 0.0016, while in Fig. 1 the amplitude is 0.0018 , and the asymmetric tunnelling regimes are observed.

Increasing further the effective nonlinearity (in graphs of Fig. 5 we have chosen $r P_{t}=2$ ) the butterfly structure enlarges and the symmetric behavior restores. Particularly, and this is clearly seen from the both graphs of the bottom panel, injecting the light in the left or right waveguide array it remain trapped there.

In order to make some predictions for realistic waveguide arrays the most convenient way is to consider discretized equation (3). Then one is able to unify all the variety of refractive index profiles via a single coupling parameter $C$. In the numerical simulations presented in Fig. $6 \mathrm{I}$ choose a coupling constant $C / 2=4 \mathrm{~mm}^{-1}$ and nonlinearity parameter is taken $\chi=6.5 \mathrm{~mm}^{-1} W^{-1}$ like in Ref. [17]. Assuming lattice spacing equal to $6 \mu m$ the tilt angle of reduced refractive index barrier is chosen $0.25 \mathrm{mrad}$ and then the samples of the length $60 \mathrm{~mm}$ will be sufficient to see the effect of asymmetric tunnelling. As it was mentioned above the main reason of appearing of such asymmetry is a nonlinearity induced butterfly structure in the reduced two degree of freedom model. In the realistic numerical simulations on discretized equation (3) the peak intensity threshold for appearing of such a structure is $\left|\mathcal{E}_{j}(Z=0)\right|_{\text {max }}^{2}=29 \mathrm{~W}$ which is experimentally easily accessible [17] quantity. Above this intensity threshold the asymmetric tunnelling behavior takes place even for fully adiabatic processes, i.e. for very small tilt angles of reduced refractive index barrier, but then long waveguide arrays will be required in order to see the effect.

Concluding it could be stated that visual observation of nonlinear extension of Landau-Zener tunnelling in optical waveguide arrays has been proposed. All analytical predictions followed from simple two degree of freedom system are completely confirmed by numerical simulations on the model equations (1) and (3).

Acknowledgements. I am very grateful to D. Christodoulides and T. Kereselidze for the useful comments. The work is supported by Georgian National Science Foundation (Grant No GNSF/STO7/4-197) and Science and Technology Center in Ukraine (Grant No $5053)$.
[1] L. D. Landau, Phys. Z. Sowjetunion, 2, 46 (1932); G. Zener, Proc. R. Soc. London A, 137, 696 (1932).

[2] L.D. Landau, E.M. Lifshitz, Quantum Mechanics, Nonrelativistic Theory, Moskow, Nauka (1989).

[3] B. P. Anderson and M. Kasevich, Science, 282, 1686
(1998).

[4] M. Cristiani, O. Morsch, J. H. Müller, D. Ciampini, E. Arimondo, Phys. Rev. A, 65, 063612 (2002).

[5] H. Sanchis-Alepuz et al, Phys. Rev. Lett. 98, 134301 (2007); L. Gutierrez et al, Phys. Rev. Lett. 97, 114301 
(2006);

[6] R. Khomeriki, S. Ruffo, Phys. Rev. Lett., 94, 113904 (2005)

[7] H. Trompeter et al, Phys. Rev. Lett., 96, 023901 (2006)

[8] F. Dreisow et al, Phys. Rev. A, 79, 055802 (2009).

[9] A. Fratalocchi, G. Assanto, Optics Express, 14, 2021 (2006).

[10] H. Trompeter et al, Phys. Rev. Lett., 96, 053903 (2006)

[11] B. Wu and Q. Niu, Phys. Rev. A, 61, 023402 (2000).

[12] J. Liu, L. Fu, B.-Y. Ou, Sh.-G. Chen, D.-I. Choi, B. Wu, Q. Niu, Phys. Rev. A, 66, 023404 (2002).

[13] D. Witthaut, E. M. Graefe, H. J. Korsch, Phys. Rev. A 73, $063609(2006)$
[14] M. Jona-Lasinio et al, Phys. Rev. Lett., 91, 230406 (2003).

[15] P. Engels, C. Atherton, Phys. Rev. Lett. 99, 160405 (2007).

[16] Yu-Ao Chen et al, arXiv:1003.4956

[17] R. Morandotti et al, Phys. Rev. Lett. 83, 4756 (1999).

[18] A.A. Sukhorukov, Y.S. Kivshar, O. Bang, C.M. Soukoulis, Phys. Rev. E, 63, 016615 (2000).

[19] S. Raghavan, A. Smerzi, S. Fantoni, S.R. Shenoy, Phys. Rev. A, 59, 620 (1999)

[20] R. Khomeriki, S. Ruffo, S. Wimberger, Europhys. Lett., 77, 40005 (2007). 